\title{
NEOPLASIAS MÚLTIPLES EN PACIENTES AFECTOS DE MELANOMA DE ÚVEA
}

\section{MULTIPLE NEOPLASMS IN PATIENTS WITH UVEAL MELANOMA}

\author{
CAMINAL JM ${ }^{1}$, MARTÍNEZ $\mathrm{J}^{2}$, ARIAS LL ${ }^{3}$, RUBIO $\mathrm{M}^{3}$, PUJOL $\mathrm{O}^{3}$, ROCA $^{3}$, ARRUGA $\mathrm{J}^{1}$
}

\section{RESUMEN}

Objetivo: Estudiar la prevalencia de neoplasias múltiples en pacientes afectos de melanoma de úvea en nuestro medio y relacionarlo con la supervivencia. Método: Para ello se ha realizado un estudio prospectivo longitudinal de prevalencia de múltiples neoplasias en pacientes diagnosticados de melanoma de úvea entre los años 1984-2005. Se han analizado entre otras las variables clínicas: edad, sexo, fecha de diagnóstico, ojo afecto, origen y tamaño tumoral, clasificación según el COMS (Collaborative Ocular Melanoma Study), fecha del último control, tiempo de seguimiento, presencia de otras neoplasias, estado sistémico actual, fecha y causa de muerte.

Resultados: Se han estudiado 305 pacientes afectos de melanoma de úvea en la Unidad de Oncologia Ocular de nuestro centro. 24 pacientes $(7,9 \%)$ presentaron en su historia médica una o más neoplasias malignas. Excluyendo las neoplasias cutáneas de origen en células basales, esta proporción fue del $7,2 \%$ (22 pacientes). No se ha encontrado diferencias estadísticamente significativas entre la edad de presentación, sexo y la localización del melanoma (cuerpo ciliar o coroides) y la presencia o no de segundas neoplasias. Cuando se analizó la proporción de pacientes con enfermedad metastásica (tan-

\begin{abstract}
Purpose: To study the prevalence of multiple neoplasms in patients affected by uveal melanoma in Spain and to relate these with survival.

Method: We carried out a longitudinal prospective study of the prevalence of multiple neoplasms in patients diagnosed to have a uveal melanoma during the years 1984-2005. The data has been analysed for the following variables: age, sex, date of diagnosis, affected eye, origin and tumoral size, classification according to COMS (Collaborative Ocular Melanoma Study), time of follow-up, presence of other neoplasms, current clinical state, date and cause of death.
\end{abstract}

Results: Three hundred and five patients affected by uveal melanoma have been studied in the Ocular Oncology Unit of our institution; 24 patients (7.9\%) had evidence in their medical reports of one or more additional neoplasms. Excluding cutaneous neoplasms originating in basal cells, this number reduced to 22 patients $(7.2 \%)$. We did not find any statistically significant differences among the presentation age, sex or localization of the melanoma (ciliary body or choroid) and the presence or absence of a second neoplasm. When we analysed the proportion of patients with metastatic disease (both alive and dead) who presented with a second neo-

\footnotetext{
Recibido: 8/6/06. Aceptado: 17/7/07.

Hospital Universitario de Bellvitge. Barcelona. España.

1 Doctor en Medicina.

2 Licenciado en Medicina. Hospital de la Santa Creu i San Pau. Barcelona. España.

3 Licenciado en Medicina.

Correspondencia:

José M. Caminal

Hospital Universitario de Bellvitge

C/. Feixa Llarga, s/n

08907 L'Hospitalet de Llobregat (Barcelona)

España

E-mail: jmcaminal@csub.scs.es
} 
to vivos como muertos) que no han presentado una segunda neoplasia $(14,3 \%)$, respecto de aquellos que si la presentaron $(40,9 \%)$, mediante el test de Chi-cuadrado, se encontró una relación estadísticamente significativa con una $\mathrm{p}=0,004$.

Conclusiones: En nuestra población de estudio, se ha observado un porcentaje de segundas neoplasias similar al descrito en otras series internacionales. No se ha encontrado una mayor proporción de segundas neoplasias según el sexo, la edad, la localización tumoral. Así como tampoco se ha observado una mayor frecuencia de determinadas segundas neoplasias. Pero sí que se ha evidenciado una mayor predisposición a desarrollar enfermedad metastásica, en los pacientes que han presentado segundas neoplasias, por lo que en estos pacientes se debe incrementar el seguimiento sistémico.

Palabras clave: Melanoma úvea, neoplasias múltiples, segundas neoplasias, factores pronósticos, tumor coroideo. plasm $(40.9 \%)$, we found a statistically significant relationship between these variables (Chi-square test, $\mathrm{p}=0.004$ ).

Conclusions: We have observed a percentage of second neoplasms similar to that described in other international studies. We did not find a larger proportion with a second neoplasm according to the sex, age, or tumoral localization, nor did we observe a higher frequency of any particular second neoplasm. We have defined a relationship between metastasic uveal melanoma, and the development of a second neoplasm, which clearly indicates a need for increased systemic follow-up in such patients (Arch Soc Esp Oftalmol 2007; 82: 535 540).

Key words: Uveal melanoma, multiple neoplasm, second neoplasm, prognostic factor, choroidal tumor.

metastásicos, el melanoma de úvea es el tumor maligno intraocular más frecuente en el adulto. El $80 \%$ de los melanomas uveales se localizan en la coroides, el $12 \%$ en el cuerpo ciliar y un $8 \%$ en el iris. Existe una predilección racial clara en el desarrollo de este tumor con una relación uno-ocho en afroamericanos con respecto a a la población caucasiana. Se han publicado algunos casos de clara incidencia familiar $(4,5)$.

Varios autores han publicado la presencia de múltiples canceres primarios en pacientes afectos de melanoma uveal $(6,7)$, lo cual sugiere un mayor riesgo para desarrollar otros tipos de neoplasias $(8,9)$. Sin embargo algunos de estos resultados no han sido confirmados por otros autores $(10,11)$.

Diferentes estudios publicados en la literatura muestran que la proporción de neoplasias secundarias en pacientes afectos de melanoma de úvea es del 8 al 14\% (12-14).

Hasta el momento no se han realizado estudios con datos de pacientes españoles, por lo que el objetivo de este trabajo es conocer la prevalencia de neoplasias múltiples en pacientes afectos de melanoma de úvea en nuestro medio, así como el tipo de neoplasias que se asocian con mayor frecuencia, e intentar determinar si este hecho condiciona un peor pronóstico vital. 


\section{SUJETOS, MATERIAL Y MÉTODOS}

Se realiza un estudio descriptivo prospectivo longitudinal de prevalencia de múltiples neoplasias malignas en una cohorte de pacientes diagnosticados de melanoma de úvea.

Se han incluido todos los pacientes diagnosticados de dicha patología en la Unidad de Oncología Ocular del Servicio de Oftalmología de nuestro centro desde el año 1984 hasta el año 2005. Se incluyeron tanto a los pacientes de las poblaciones del área de influencia de este hospital, como a los que fueron remitidos de otras zonas de España.

Para cada paciente se registraron la edad, sexo, fecha de diagnóstico del melanoma de úvea, localización del tumor, estado vital al final del estudio, causa de muerte, presencia de otras neoplasias antes, en el momento del diagnóstico o durante el seguimiento. Se clasificaron las lesiones como sincrónicas si se diagnosticaban en un intervalo de 6 meses antes o después del diagnóstico del melanoma de úvea y como metacrónicas si este diagnóstico se realizaba en un intervalo superior a 6 meses.

Se realizó un análisis estadístico con el paquete informático SPSS versión 10.0 (SPSS por Windows, SPSS Inc, Chicago, USA) y se usaron los estadísticos Chi-cuadrado o Fische, según convino.

\section{RESULTADOS}

Se diagnosticaron un total de 305 pacientes afectos de melanoma de úvea, en la Unidad de Oncologia Ocular de nuestro centro, durante el período del estudio, de los cuales $170(55,7 \%)$ eran mujeres y 135 $(44,3 \%)$ eran varones. El rango de edad en el momento de la primera visita oscilaba entre los 25 y los 89 años, con una media de 58,6 años. En el 51\% de los pacientes el tumor afectaba al ojo derecho y en el $49 \%$ al izquierdo. En 256 casos $(83,9 \%)$ el melanoma afectaba a la coroides, en 40 casos $(13,1 \%)$ al cuerpo ciliar y en siete casos $(2,3 \%)$ al iris.

El seguimiento medio de nuestra serie ha sido de 1144.6 días (IC 1041-1248.2). Del total de 305 pacientes estudiados, 24 de ellos $(7,9 \%)$ presentaban en su historial médico, una o más neoplasias malignas. Cuando excluimos las neoplasias cutáneas de origen en células basales, esta proporción fue del 7,2\% (22 pacientes).

De los 24 pacientes afectos de neoplasias malignas múltiples, en 18 casos $(81,8 \%)$ el melanoma afectaba a la coroides, en cuatro casos $(18,2 \%)$ al cuerpo ciliar y en ningún caso al iris. La edad media de los pacientes afectos de neoplasias múltiples fue de 63,45 años, con un rango de 30,79 a 85,24 años, sin evidenciarse diferencias entre sexos. La proporción de mujeres y de hombres era del 54,2\% (13 pacientes) y del 45,8\%(11 pacientes) respectivamente.

En cuanto al número de neoplasias presentes teniendo en cuenta el melanoma de úvea, fue de dos neoplasia en 21 pacientes $(87,5 \%)$, tres neoplasias en dos pacientes $(8,3 \%)$ y de cuatro neoplasias en un paciente $(4,1 \%)$.

En los 21 pacientes que presentaban dos neoplasias múltiples, se encontraron cuatro casos en que la neoplasia era sincrónica (19\%). En el resto de pacientes el diagnóstico de las neoplasias fue metacrónico (81\%).

No se han encontrado diferencias estadísticamente significativas entre la edad de presentación, sexo y la localización del melanoma (cuerpo ciliar o coroides) y la presencia o no de segundas neoplasias.

Cuando se analizó el tipo de segunda neoplasia que presentaban los pacientes de nuestra serie, se encontró que el cáncer más frecuente fue el adenocarcinoma de mama $(17,8 \%)$, seguido en la misma proporción del adenocarcinoma de próstata, colon, melanoma cutáneo, carcinoma de vejiga, carcinoma de laringe, carcinoma escamoso cutáneo y carcinoma basocelular $(7,1 \%$ cada uno de ellos) (tabla I). Se analizan los datos según el sexo de los pacientes, en

\section{Tabla I. Localización de segundas neoplasias diagnosticadas en 24 pacientes afectos de melanoma de úvea}

\begin{tabular}{lcr}
\hline Tipo neoplasia & N. $^{\text {o }}$ neoplasias & Porcentaje \\
\hline Carcinoma de mama & 5 & $17,8 \%$ \\
Adenocarcinoma de próstata & 2 & $7,1 \%$ \\
Adenocarcinoma colon/sigma & 2 & $7,1 \%$ \\
Melanoma cutáneo & 2 & $7,1 \%$ \\
Carcinoma de vejiga & 2 & $7,1 \%$ \\
Carcinoma escamoso de laringe & 2 & $7,1 \%$ \\
Carcinoma escamoso de piel & 2 & $7,1 \%$ \\
Carcinoma basocelular & 2 & $7,1 \%$ \\
Carcinoma escamoso de pulmón & 1 & $3,5 \%$ \\
Carcinoma renal & 1 & $3,5 \%$ \\
Hemangioblastoma cerebeloso & 1 & $3,5 \%$ \\
Macroglobulinemia de Waldenström & 1 & $3,5 \%$ \\
Carcinoma de esófago & 1 & $3,5 \%$ \\
Linfoma no Hodgkin & 1 & $3,5 \%$ \\
Limfoma cutáneo de células T & 1 & $3,5 \%$ \\
Carcinoma escamoso de pene & 1 & $3,5 \%$ \\
Carcinoma escamoso de base & 1 & $3,5 \%$ \\
$\quad$ de la lengua & & \\
Total & 28 & $100 \%$ \\
\hline \hline
\end{tabular}


las mujeres la segunda neoplasia más frecuente fue el carcinoma de mama y el adenocarcinoma de colon, y en los hombres el adenocarcinoma de próstata, el carcinoma de vejiga urinaria y el carcinoma escamoso de laringe (tablas II y III).

Cuando se analizaron la proporción de pacientes con enfermedad metastásica (tanto vivos como muertos) que no han presentado una segunda neoplasia $(14,3 \%)$, respecto de aquellos que si la presentaron (40,9\%), mediante el test de Chi-cuadrado, se encontró una relación estadísticamente significativa con una $\mathrm{p}=0,004$ (fig. 1 ).

\section{DISCUSIÓN}

El melanoma de úvea es un tumor poco prevalente con una incidencia anual de seis nuevos casos por millón de habitantes en los Estados Unidos (3). Aún

Tabla II. Localización de segundas neoplasias diagnosticadas en 13 mujeres afectas de melanoma de úvea

Tipo neoplasia en mujeres

Carcinoma de mama

Adenocarcinoma colon/sigma

Carcinoma renal

Melanoma cutáneo

Hemangioblastoma cerebeloso

Macroglobulinemia de Waldenström

Carcinoma escamoso cutáneo

Carcinoma basocelular

Total

N. ${ }^{\circ}$ neoplasias Porcentaje

Tabla III. Localización de segundas neoplasias diagnosticadas en 11 hombres afectos de melanoma de úvea

Tipo neoplasia en hombres

N. ${ }^{\circ}$ neoplasias Porcentaje

\begin{tabular}{llr}
\hline Adenocarcinoma de próstata & 2 & $13,3 \%$ \\
Carcinoma de vejiga & 2 & $13,3 \%$ \\
Carcinoma escamoso de laringe & 2 & $13,3 \%$ \\
Carcinoma escamoso de pulmon & 1 & $6,6 \%$ \\
Carcinoma de esófago & 1 & $6,6 \%$ \\
Linfoma no Hodgkin & 1 & $6,6 \%$ \\
Melanoma cutáneo & 1 & $6,6 \%$ \\
Limfoma cutáneo de células T & 1 & $6,6 \%$ \\
Carcinoma escamoso de pene & 1 & $6,6 \%$ \\
Carcinoma escamoso de base & 1 & $6,6 \%$ \\
$\quad$ de la lengua & & $6,6 \%$ \\
Carcinoma escamoso cutáneo & 1 & $6,6 \%$ \\
Carcinoma basocelular & 1 & $100 \%$ \\
& 15 &
\end{tabular}

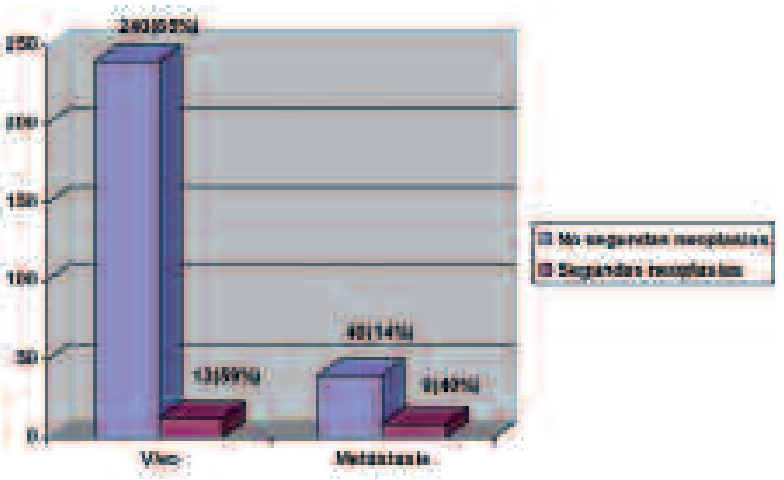

Fig. 1: Proporción de pacientes con enfermedad metastásica vivos o muertos en los cuales no se ha diagnosticado una segunda neoplasia (14\%), respecto a los pacientes que si la han presentado (40\%). Chi-cuadrado $p=0,004$.

así es el tumor intraocular primario maligno más frecuente. Muchos aspectos como la epidemiología, la patogénesis o el tratamiento de esta neoplasia continúan siendo controvertidos. Hemos realizado este estudio para investigar la epidemiología de esta neoplasia, en concreto queríamos conocer la prevalencía de otras neoplasias asociadas, en los pacientes afectos de melanoma de úvea diagnosticados en la Unidad de Oncología Ocular de nuestro centro. No existen publicaciones en nuestra comunidad que describan aspectos epidemiológicos de pacientes afectos de melanoma de úvea. Además el hecho de intentar buscar asociaciones entre diferentes cánceres puede permitir demostrar causas potencialmente similares e identificar poblaciones que presentan un peor pronóstico vital.

Nuestro porcentaje de pacientes con más de una neoplasia fue del 7,2\%, valor similar al descrito en el estudio COMS (Collaborative ocular melanoma study), que fue del 7,7\% (excluyendo en ambos casos a los tumores cutáneos de células basales). Este porcentaje no difiere mucho de la probabilidad de desarrollar un cáncer invasivo entre los 40 y 59 años de edad tanto para hombres y mujeres que es del 8 al 9\%, en los Estados Unidos basándose en datos de 1998 al 2000. Estos porcentajes no son completamente comparables puesto que en nuestro estudio hemos tabulado todas las segundas neoplasias que presentaron los pacientes antes, durante o después del diagnóstico de melanoma de úvea, y en el estudio del COMS sólo se consideraron las neoplasias aparecidas después de su diagnóstico (15). En otros estudios la prevalencia de segunda neopla- 
sias en pacientes con melanoma uveal oscila entre el $11 \%$ al $14 \%(12-14)$.

La localización de las segundas neoplasias más frecuente fue el carcinoma de mama, seguido del adenocarcinoma de próstata, colon, melanoma cutáneo, carcinoma de vejiga, laringe y pulmón. En otros estudios las localizaciones más frecuentes son la mama y la próstata (15).

Algunos autores han encontrado una mayor prevalencia de neoplasia ginecológica (cáncer uterino y cervical) entre las mujeres con melanoma de úvea respecto a un grupo control (13). En un estudio danés sobre 2.018 pacientes afectos de melanoma de úvea, encontraron un mayor riesgo global de segundas neoplasias en hombres respecto de las mujeres, y tanto unos como otros presentaban un riesgo superior de desarrollar cáncer primario hepático, no relacionado con enfermedad metastásica (12). En nuestra serie no hemos evidenciado ninguna de estas asociaciones, así como tampoco hemos observado una mayor prevalencia de un tipo concreto de segunda neoplasia, tanto en hombres como en mujeres, al igual que en otros trabajos (14).

Sorprende la presentación de más de un segundo tumor además del melanoma en casi el 1\% de nuestra serie (tres pacientes), hecho que nos hace pensar en una predisposición individual a la génesis de neoplasias, probablemente de causa genética. De hecho se ha descrito esta predisposición al desarrollo de múltiples neoplasias, siendo identificados más de 30 genes que están relacionados con una mayor susceptibilidad al cáncer (1). La presencia de segundas neoplasias puede asociarse también a tratamientos que potencialmente son carcinogénicos, como la radioterapia externa (telerradioterapia) o la quimioterapia, sin embargo en nuestros pacientes esta posibilidad es muy remota, puesto que habitualmente son tratados mediante fotocoagulación láser, cirugía o radioterapia localizada (braquiterapia).

No hemos encontrado diferencias estadísticamente significativas entre la edad de presentación, sexo y la localización del melanoma (cuerpo ciliar o coroides) y la presencia o no de segundas neoplasias.

Hemos observado una mayor presencia de enfermedad metastásica entre aquellos pacientes con segundas neoplasias que entre aquellos que no las presentaron, siendo esta diferencia estadísticamente significativa ( $\mathrm{p}=0,004$. Test de Chi-cuadrado). Esta asociación representa un peor pronóstico vital para aquellos pacientes que han desarrollado una segunda neoplasia antes del diagnóstico del melanoma de úvea o que les aparece durante su seguimiento y probablemente obedece a una mayor predisposición genética para el desarrollo del cáncer.

Las limitaciones de este estudio vienen dadas porque la prevalencia de segundas neoplasias podrían ser superiores a las registradas, puesto que los historiales médicos pueden estar incompletos o bien el paciente no nos explica en la primera visita o en el seguimiento el episodio canceroso que presentó o ha presentado. Además puesto que las localizaciones de las segundas neoplasias son en puntos distantes al ojo, se hace difícil el diagnóstico precoz de estas lesiones en el screening sistémico standard para el estudio metastásico en los pacientes afectos de melanoma de úvea.

Tampoco hemos podido establecer relaciones sobre una mayor prevalencia de determinadas neoplasias en nuestra población de pacientes.

Como conclusión podemos afirmar que en nuestra población de estudio, hemos observado un porcentaje de segundas neoplasias similar al descrito en otras series internacionales. No hemos encontrado una mayor proporción de segundas neoplasias según el sexo, la edad, la localización tumoral. Así como tampoco hemos observado una mayor frecuencia de determinadas segundas neoplasias. Pero sí que hemos evidenciado una mayor predisposición a desarrollar enfermedad metastásica, en los pacientes que han presentado segundas neoplasias, por lo que en estos pacientes debemos incrementar nuestro seguimiento sistémico.

\section{BIBLIOGRAFÍA}

1. Knudson AG. Karnofsky Memorial Lecture. Hereditary cancer: theme and variations. J Clin Oncol 1997; 15: 3280-328.

2. Dong $C$, Hemminski $K$. Second primary neoplasm in 633,964 cancer patients in Sweden, 1958-1996. Int J Cancer 2001; 93: 155-161.

3. Egan KM, Seddon JM, Glynn RJ, Gragoudas ES, Albert DM. Epidemiologic aspects of uveal melanoma. Surv Ophthalmol 1988; 32: 239-251.

4. Kodjikian L, Nguyen K, Lumbroso L, Gauthier-Villars, Chauvel P, Planchu $H$ et al.Familial uveal melanoma: report on two families and a review of the literature. Acta Ophthalmol Scand 2003; 81: 389-395.

5. Krygier $G$, Lombardo K, Vargas C, Alvez, J, Costa R, Ros $M$ et al. Familial uveal melanoma: report on three sibling cases. Br J Ophthalmol 2001; 85: 1007-1008.

6. Rednam KR, Jampol LM, Levine RA, Goldberg MF. Uveal melanoma in association with multiple malignancies.A case report and review. Retina 1981; 1: 100-106. 
7. Henkind P, Roth M. Breast carcinoma and concurrent uveal melanoma. Am J Ophthalmol 1971; 71: 199-203.

8. Shors A, Iwamoto S, Doody DR, Weiss NS. Relationship of uveal and cutaneous malignant melanoma in persons with multiple primary tumors. Int J Cancer 2002; 102: 266268.

9. Bergman L, Nilsson B, Ragnarsson-Olding B, Seregard S. Uveal melanoma: a study on incidence of additional cancers in the Swedish population. Invest Ophthalmol Vis Sci 2006; 47: 72-77.

10. Holly EA, Aston DA, Ahn DK, Kristiansen JJ, Char DH. No excess prior cancer $i$ n patients with uveal melanoma. Ophthalmology 1991; 98: 608-611.

11. Lischko AM, Seddon JM, Gragoudas ES, Egan KM, Glynn $R J$. Evaluation of prior primary malignancy as a determinat of uveal melanoma. A case-control study. Ophthalmology 1989; 96: 1716-1721.
12. Swerdlow AJ, Storm HH, Sasieni PD. Risk of second primary malignancy in patients with cutaneous and ocular melanoma in denmark, 1943-1989. Int J Cancer 1995; 61: 773-779.

13. Turner BJ, Siatkowski RM, Augsburger JJ, Shields JA, Lustbader E, Mastrangelo MJ. Other cancers in uveal melanoma patients and their families. Am J Ophthalmol 1989; 107: 601-608.

14. Callejo SA, Al-Khalifa S, Orzal PC, Edelstein C, Burnier MN Jr. The risk of other primary cancer in patients with uveal melanoma: a retrospective cohort study of a Canadian population.Can J Ophthalmol 2004; 39: 397-402.

15. Diener-West M, Reynolds SM, Agugliaro DJ, Cadwell R, Cumming K, Earle DJ, et al. Second primary cancers after enrollment in the COMS trials for treatment of choroidal melanoma: COMS report No. 25. Arch Ophthalmol 2005; 123: 601-604. 